\title{
HMGB1/PI3K/Akt/mTOR Signaling Participates in the Pathological Process of Acute Lung Injury by Regulating the Maturation and Function of Dendritic Cells
}

\section{OPEN ACCESS}

Edited by:

Betty Diamond,

Feinstein Institute for Medical

Research, United States

Reviewed by:

David L. Perkins,

University of Illinois at Chicago,

United States

Antonella Naldini,

University of Siena, Italy

${ }^{*}$ Correspondence:

You Shang

1686406592@qq.com

Specialty section:

This article was submitted to

Inflammation,

a section of the journal

Frontiers in Immunology

Received: 21 March 2020

Accepted: 06 May 2020

Published: 19 June 2020

Citation:

Li R, Zou X, Huang H, Yu Y, Zhang $H$,

Liu P, Pan S, Ouyang $Y$ and Shang $Y$ (2020) HMGB1/PI3K/AKt/mTOR

Signaling Participates in the

Pathological Process of Acute Lung

Injury by Regulating the Maturation and Function of Dendritic Cells.

Front. Immunol. 11:1104.

doi: 10.3389/fimmu.2020.01104

\begin{abstract}
Ruiting $\mathrm{Li}^{1}$, Xiaojing Zou ${ }^{1}$, Haiyan Huang ${ }^{1}$, Yuan $\mathrm{Yu}^{1}$, Hongmei Zhang ${ }^{2}$, Pei Liu ${ }^{3}$, Shangwen Pan ${ }^{1}$, Yaqi Ouyang ${ }^{1}$ and You Shang ${ }^{1 *}$
\end{abstract}

${ }^{1}$ Department of Critical Care Medicine, Institute of Anesthesia and Critical Care Medicine, Union Hospital, Tongji Medical College, Huazhong University of Science and Technology, Wuhan, China, ${ }^{2}$ Department of Emergency, Shenzhen People's Hospital, First Affiliated Hospital of Southern University of Science and Technology, Second Clinical Medical College of Jinan University, Shenzhen, China, ${ }^{3}$ Shenzhen Key Laboratory of Respiratory Diseases, Department of Respiratory and Critical Care Medicine, Shenzhen Institute of Respiratory Diseases, Shenzhen People's Hospital, First Affiliated Hospital of Southern University of Science and Technology, Second Clinical Medical College of Jinan University, Shenzhen, China

Background: High-mobility group box 1 protein (HMGB1) was identified as a highly conserved DNA binding nuclear protein, which participates in the processes of acute lung injury (ALI). HMGB1 binds to its specific receptors not only to activate the nuclear factor (NF)-кB and mitogen-activated protein kinase (MAPK) pathways but also to regulate the activation of the phosphatidylinositol 3'-kinase/protein kinase B/mammalian target of the rapamycin (PI3K/AKT/mTOR) pathway. Mature dendritic cells (DCs) regulate acute lung inflammation and pathological injury in ALI. In addition, studies have shown that the activation of the PI3K/AKT/mTOR signaling pathway may regulate the function and maturation of DCs.

Objective: Therefore, we speculate that HMGB1/PI3K/Akt/mTOR signaling participates in regulating the pathological process of $\mathrm{ALI}$ by regulating the maturation and function of DCs.

Methods: Anti-HMGB1 antibody, rHMGB1, or LY294002 (PI3K inhibitor) was administered in a murine model of lipopolysaccharide (LPS)-induced ALI. For in vitro studies, generated bone marrow-derived dendritic cells (BMDCs) primed by LPS were stimulated with the same reagents. The effects of these different treatments were observed on the expression of PI3K, AKT, and mTOR and on the function of DCs.

Results: HMGB1 upregulated the expression of PI3K, Akt, and mTOR mRNA and phosphorylated proteins in BMDCs. The HMGB1/PI3K/Akt/mTOR signaling pathway induced the maturation and antigen-presenting ability of lung DCs, mediated the percentage of myeloid DCs (mDCs), and enhanced the adhesion and chemotactic ability of lung DCs.

Conclusions: HMGB1/PI3K/Akt/mTOR signaling participates in the pathological process of ALI by regulating the maturation and functions of DCs.

Keywords: HMGB1, PI3K/Akt/mTOR signaling, acute lung injury, lipopolysaccharide, dendritic cell 


\section{INTRODUCTION}

Acute lung injury (ALI)/acute respiratory distress syndrome (ARDS) is a complex clinical syndrome characterized by persistent hypoxemia due to pulmonary interstitial edema, damage and disruption of the alveolar-capillary barrier, and widespread inflammation in the lung $(1,2)$. Although the clinical treatment and management of ALI/ARDS have improved, it still has high morbidity and mortality in the intensive care unit (3). Previous studies have shown that an excessive inflammatory response storm plays a vital role in the pathological process of ALI/ARDS (1). Therefore, elucidation of molecular and cellular mechanisms associated with inflammation in ALI may be helpful in identifying new therapeutic targets.

High-mobility group box 1 protein (HMGB1) was identified as a highly conserved DNA binding nuclear protein and participates in the processes of replication, recombination, transcription, and DNA repair. HMGB1 is reported to contribute to inflammatory dysfunction in sepsis and ALI, and the levels of HMGB1 in the plasma and tissue were significantly increased in a mouse model of lipopolysaccharide (LPS)-induced mouse model of ALI $(4,5)$. Increasing evidence supports the role of HMGB1 as a regulator of ALI. The downstream pathways of HMGB1 may lead to neutrophil infiltration, injury of lung tissue, inflammatory cytokine release, and the development of ALI. HMGB1 binds to its specific receptors, including the receptor for advanced glycation end products, toll-like receptor (TLR)2 and TLR4, in turn activating the nuclear factor (NF)-КB and mitogen-activated protein kinase (MAPK) pathways, which mediate inflammatory molecules such as TNF$\alpha$, IL-1 $\beta$, IL-18, and IL-6 (6-8). HMGB1 also induces an inflammatory response through the phosphatidylinositol $3^{\prime}$ kinase/protein kinase $\mathrm{B} /$ mammalian target of the rapamycin (PI3K/AKT/mTOR) pathway (9). Previous studies have shown that HMGB1 inhibitors or HMGB1 siRNA effectively inhibited the activation of the TLR4/NF- $\mathrm{KB}$ and PI3K/AKT/mTOR pathways $(9,10)$. Previous research has shown that the PI3K/Akt/mTOR pathway regulated multiple physiological activities such as cell proliferation, autophagy, and apoptosis (11, 12). In addition, the PI3K/Akt/mTOR pathway has an important role in pulmonary inflammation and pathological progression of ALI $(13,14)$. Thus, HMGB1 induces an inflammatory response in ALI through the PI3K/AKT/mTOR pathway.

Dendritic cells (DCs), the most prominent antigen-presenting cells, play a key role in initial and adaptive immune responses and are ideally positioned to serve a priming and central

\footnotetext{
Abbreviations: ALI, acute lung injury; LPS, lipopolysaccharide; ARDS, acute respiratory distress syndrome; HMGB1, high mobility group box1; BALF, bronchoalveolar lavage fluid; DCs, dendritic cells; MNCs, lung mononuclear cells; NF-кB, nuclear factor kappa B; TLR, toll-like receptor; BMDCs, bone marrow-derived dendritic cells; MAPK, mitogen-activated protein kinase; cDCs, conventional DCs; RT-qPCR, real-time reverse-transcriptase polymerase chain reaction; ELISA, enzyme-linked immunosorbent assay; IL, interleukin; TNF, tumor necrosis factor; MCP, monocyte chemotactic protein; cDNA, complementary DNA; GAPDH, glyceraldehyde 3-phosphate dehydrogenase; SDS-PAGE, sodium dodecyl sulfate-polyacrylamide gel electrophoresis; PVDF, polyvinylidene fluoride; ICAM, intercellular adhesion molecule; mDCs, myeloid dendritic cells; pDCs, plasmacytoid dendritic cells
}

role in the immune response during inflammation $(15,16)$. Previous studies have shown that the number of mature conventional DCs (cDCs) in lung tissue during ALI is significantly increased, and the maturation of pulmonary DCs regulate acute lung inflammation and pathological injury (17, 18). Therefore, DCs play an important role in the pathological progress of ALI, and regulating the function and maturation of DCs may have great clinical significance for the treatment of ALI.

Studies have shown that promoting the activation of $\mathrm{PI} 3 \mathrm{~K} / \mathrm{AKT} / \mathrm{mTOR}$ signaling regulates the function of immune cells including DCs $(19,20)$. Moreover, the activation of the PI3K/Akt/mTOR pathway participates in sepsis-induced ALI $(11,13,21)$. Thus, we speculated that HMGB1/PI3K/Akt/mTOR signaling participates in regulating the pathological process of ALI by regulating the maturation and function of DCs.

\section{MATERIALS AND METHODS}

\section{Mice}

Male C57BL/6 mice (6-8 weeks old, 20-22 g) were obtained from the Experimental Animal Centre of Hubei province (Wuhan, China). The mice were maintained in the animal laboratory of the Experimental Animal Center of Hubei province under standard laboratory conditions for 1 week prior to the experiments. The mice were kept under specific pathogen-free conditions at $25^{\circ} \mathrm{C}$ in a 12 -h light/dark cycle, with a humidity of $45-55 \%$ in a ventilated cage. All experimental procedures were performed in accordance with the requirements of the Institutional Animal Care and Use Committee at Huazhong University of Science and Technology (Wuhan, China).

\section{Experimental Protocol for the ALI Murine Model}

All mice were divided into the following groups using the randomized grouping method ( $n=4-6$ per group): control group, LPS group, LPS+anti-HMGB1 group, LPS+rHMGB1 group, LY294002 (PI3K inhibitor) positive control group, and LPS+LY294002 group. The ALI murine model was induced by intraperitoneal (i.p.) injection of LPS as described previously (22). Anti-HMGB1 or rHMGB1 was administered as previously described (22). The LY294002 intervention group was injected with $1 \mathrm{mg} / 25 \mathrm{~g}$ LY294002 via the tail vein $2 \mathrm{~h}$ after LPS injection (23). All experimental mice were sacrificed using cervical dislocation $24 \mathrm{~h}$ after receiving the LPS challenge, and lung tissues and bronchoalveolar lavage fluid (BALF) were extracted for further analysis.

\section{Collection of BALF}

We collected BALF as described in detail in a previous study (24). 
TABLE 1 | Primer sequences and product sizes.

\begin{tabular}{|c|c|c|}
\hline Gene name & Primer sequences $\left(5^{\prime}-3^{\prime}\right)$ & $\begin{array}{l}\text { Product } \\
\text { size (bp) }\end{array}$ \\
\hline $\begin{array}{l}\text { PI3K Forward: } \\
\text { Reversed: }\end{array}$ & $\begin{array}{l}\text { 5'- ACACCACGGTTGGGACTATGG -3' } \\
\text { 5'- GGCTACAGTAGTGGGCTTGG -3' }\end{array}$ & 140 \\
\hline $\begin{array}{l}\text { Akt Forward: } \\
\text { Reversed: }\end{array}$ & $\begin{array}{l}\text { 5'- TGGGTCAAGGAACAGAAGCA -3' } \\
5^{\prime} \text { - TCACACTGACCACTGACACA -3' }\end{array}$ & 111 \\
\hline $\begin{array}{l}\text { mTOR Forward: } \\
\text { Reversed: }\end{array}$ & $\begin{array}{l}\text { 5'-CGGGACTCTTIACACTGCG-3' } \\
\text { 5'-CCTTCAGGCTCAACCAACA-3' }\end{array}$ & 82 \\
\hline $\begin{array}{l}\text { GAPDH Forward: } \\
\text { Reversed: }\end{array}$ & $\begin{array}{l}\text { 5'-TGTGTCCGTCGTGGATCTGA-3' } \\
\text { 5'-TTGCTGTTGAAGTCGCAGGAG-3' }\end{array}$ & 150 \\
\hline
\end{tabular}

(a) PI3K, Phosphatidylinositol 3'-kinase; (b) Akt, Protein kinase B; (c) mTOR, Mammalian target of rapamycin; (d) GAPDH, Glyceraldehyde-3-phosphate dehydrogenase.

\section{Generation of Bone Marrow-Derived Dendritic Cells (BMDCs)}

BMDCs were generated as previously described (24). The obtained BMDCs were stimulated with or without $1 \mu \mathrm{g} / \mathrm{ml}$ LPS (Sigma Aldrich, St. Louis, MO, USA), anti-HMGB1 $(10 \mu \mathrm{g} / \mathrm{ml})$ (22), rHMGB1 $(50 \mu \mathrm{g} / \mathrm{ml})$ (22), or LY294002 (25 $\mu \mathrm{M})$ (25) for $24 \mathrm{~h}$. BMDCs and cell supernatants were collected for subsequent real-time reverse-transcriptase polymerase chain reaction (RT-qPCR) and enzyme-linked immunosorbent assay (ELISA) analyses.

\section{Cytokine Analysis}

Concentrations of interleukin (IL)-12p40, tumor necrosis factor (TNF)- $\alpha$, IL-6, IL-18, IL-1 $\beta$, and monocyte chemotactic protein (MCP)-1 secreted by DCs from each sample were determined by ELISA according to the manufacturer's instructions (eBioscience, San Diego, CA, USA). Cytokine concentrations are expressed as $\mathrm{pg} / \mathrm{ml}$.

\section{RNA Extraction and RT-qPCR}

Total RNA was extracted from the right lung tissue and BMDCs by a Trizol reagent (Invitrogen/Thermo Fisher Scientific Inc., Carlsbad, CA, USA), and the RNA was reverse-transcribed into complementary DNA (cDNA) using a ReverTra Ace qPCR RT kit (Toyobo CO., LTD., Tokyo, Japan) according to the manufacturer's protocol.

RT-qPCR assay on the samples was carried out using the SYBR Premix Ex Taq ${ }^{\mathrm{TM}}$ (Takara Bio Inc., Otsu, Japan) as previously described $(24,26)$. RT-qPCR data were analyzed by QuantStudio 6 Flex (ABI Life Technology, USA). The $2^{\Delta \Delta C t}$ method was used to evaluate the relative expression of each target gene after normalization by glyceraldehyde 3-phosphate dehydrogenase (GAPDH). Primer sequences for each target gene are listed in Table 1.

\section{Western Blot Analysis}

BMDCs were harvested at $24 \mathrm{~h}$ after stimulation with LPS and frozen at $-80^{\circ} \mathrm{C}$. Total protein was extracted using a lysis buffer (Beyotime Institute of Biotechnology, Haimen, China). Protein concentrations were determined by BCA Protein
Assay Kit (Beyotime Biotechnology, Shanghai, China). Protein (40 $\mu \mathrm{g} /$ well) was separated via $10 \%$ sodium dodecyl sulfatepolyacrylamide gel electrophoresis (SDS-PAGE) and transferred to polyvinylidene fluoride (PVDF) membranes (Millipore Corp., Billerica, MA, USA). Membranes were blocked with 5\% non-fat milk in Tris-buffered saline containing Tween 20 (TBST) for $2 \mathrm{~h}$ at room temperature, then incubated with primary antibodies (p-PI3K p85, p-Akt T308, p-mTOR, Abcam, Cambridge, UK) diluted in a blocking solution $(1: 1,000)$ overnight at $4^{\circ} \mathrm{C}$, and finally treated with diluted horseradish peroxidase (HRP)-conjugated secondary antibody (1:50,000, BOSTER Biological Technology Co., Wuhan, Chine) at $37^{\circ} \mathrm{C}$ for $2 \mathrm{~h}$. The immunoreactive bands were analyzed using the BandScan 5.0 (Glyko, Novato, CA, USA) gel imaging software.

\section{Flow Cytometry}

To determine the phenotype of DCs in lung tissue and BMDCs, prepared lung mononuclear cells (MNCs) and BMDCs were suspended in a FACS buffer at $2 \times 10^{6}$ cells $/ \mathrm{ml}$. DCs express the marker CD11c but not the marker F4/80 on the cell surface; therefore, DCs were gated as $\mathrm{CD} 11 \mathrm{c}^{+} / \mathrm{F} 4 / 80^{-}$cells using the $\mathrm{PE}-\mathrm{CD} 11 \mathrm{c}$ antibody (eBioscience, San Diego, CA, USA) and the APC-Cy7-F4/80 antibody (BioLegend Inc., San Diego, CA, USA). Next, the cells were stained with the following antibodies: FITCMHCII, FITC-CD80, FITC-CD40, FITC-B220, FITCICAM-1 (eBioscience, San Diego, CA, USA), APC-CD86, APC-CCR7, APC-CD11b (Biolegend Inc., San Diego, CA, USA). All cells were analyzed by flow cytometry (FACSAria ${ }^{\mathrm{TM}}$ III, BD Biosciences, USA).

\section{Statistical Analysis}

The data are presented as the mean \pm standard deviation (SD). All experiments were repeated at least three times. One-way analysis of variance was used to compare differences. Statistical analysis was carried out using the GraphPad Prism 6 software (GraphPad Software Inc., San Diego, CA, USA) and the SPSS 22.0 software (IBM SPSS, Chicago, IL, USA). Differences were considered significant at $p<0.05 .{ }^{*} p<0.05$; ${ }^{* *} p<0.01$; and ${ }^{* * *} p$ $<0.001$.

\section{RESULTS}

\section{HMGB1 Activated PI3K/Akt/mTOR Signal Pathway in BMDCs}

Previous studies have shown that HMGB1 regulates the $\mathrm{PI} 3 \mathrm{~K} / \mathrm{Akt} / \mathrm{mTOR}$ pathway in myocardial ischemia reperfusion injury and the ALI mouse model $(9,27)$. We examined the effect of HMGB1 on PI3K/Akt/mTOR signaling in BMDCs following administration with rHMGB1 or anti-HMGB1 by Western blot and RT-qPCR analysis. HMGB1-mediated PI3K/Akt/mTOR pathway activation was assessed by detecting p-PI3K, p-Akt, and p-mTOR protein and PI3K, Akt, and mTOR mRNA expression levels. Western blot and RT-qPCR analysis revealed a remarkable increase in the expression of p-PI3K, p-Akt, and p-mTOR protein 
A

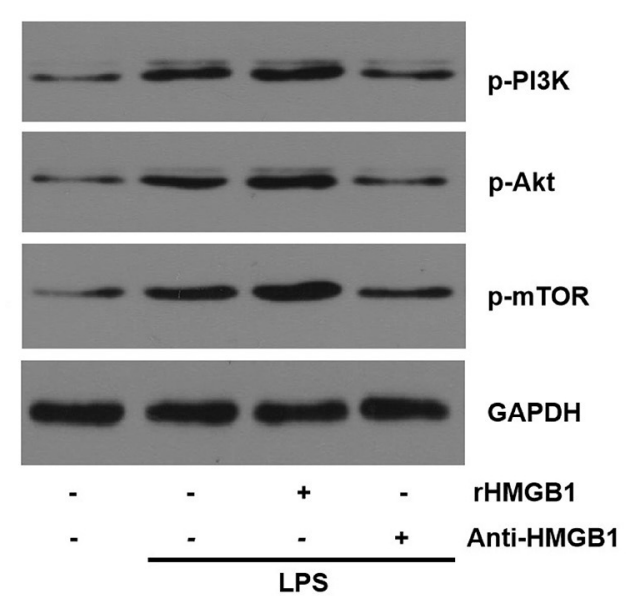

B

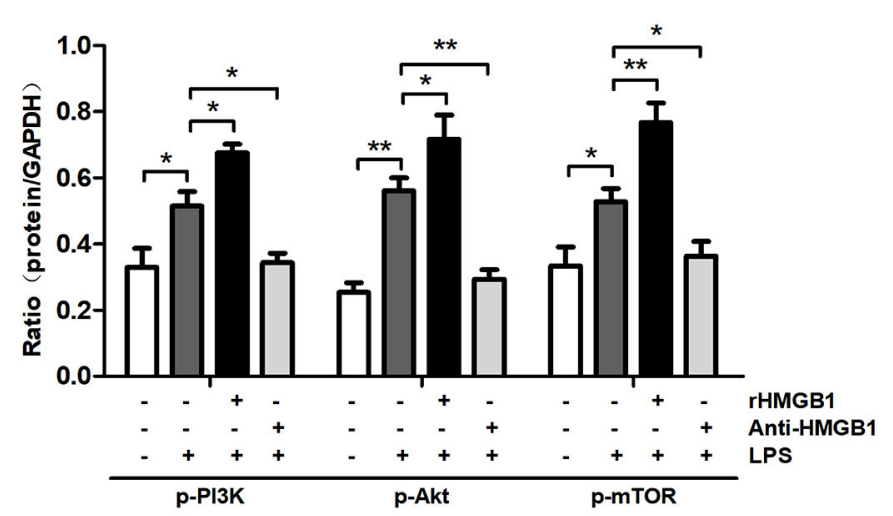

c

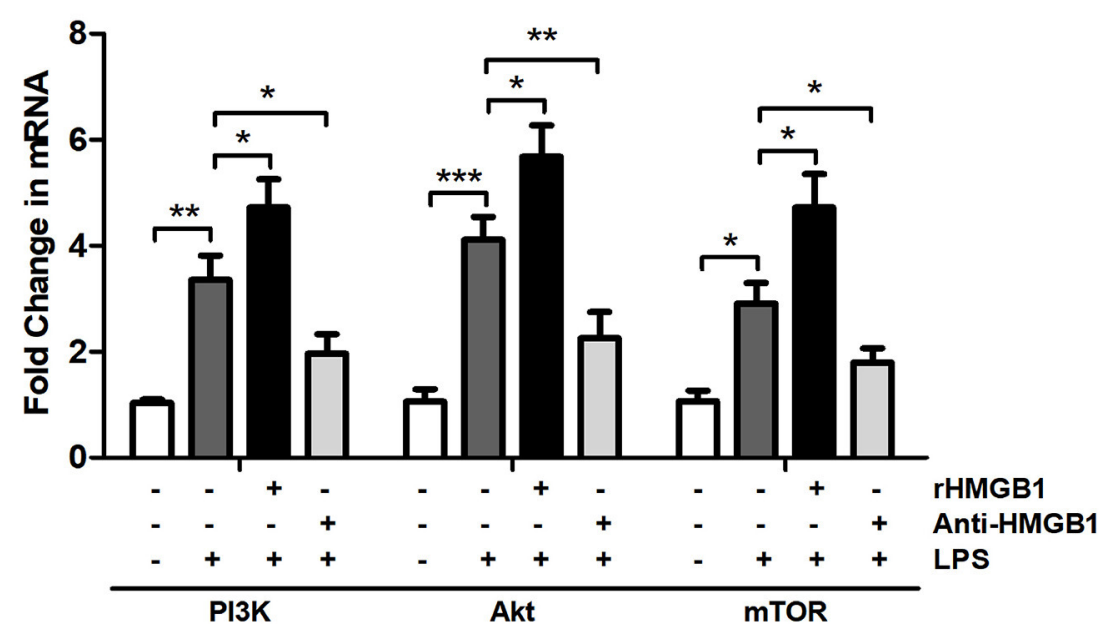

FIGURE 1 | Anti-HMGB1 and rHMGB1 regulated the expression of the PI3K/Akt/mTOR signaling pathway in BMDCs. (A,B) Expression of p-PI3K, p-Akt, and p-mTOR in BMDCs of different groups was measured by Western blot analysis. GAPDH served as a loading control. (C) Expression of PI3K, Akt, and mTOR mRNA in BMDCs of different groups was measured by RT-PCR. GAPDH served as the housekeeping gene. ${ }^{*} p<0.05,{ }^{* *} p<0.01,{ }^{\star * *} p<0.001$.

(Figures 1A,B) and PI3K, Akt, and mTOR mRNA (Figure 1C) in LPS-primed BMDCs. Moreover, rHMGB1 treatment further increased the expression of these proteins and transcripts, but this increase was attenuated by anti-HMGB1 (Figures 1A-C). These results suggest that HMGB1 is an activator of the $\mathrm{PI} 3 \mathrm{~K} / \mathrm{Akt} / \mathrm{mTOR}$ pathway in DCs.

\section{HMGB1 Induced the Maturation of DCs in vivo and in vitro}

The activation and maturation of DCs are characterized by the expression of MHCII and various costimulatory molecules on their surface and the secretion of related inflammatory cytokines $(28,29)$. To determine whether HMGB1 affects the maturation and function of DCs, we observed the effect of HMGB1 on MHCII, CD80, CD86, and CD40 of lung DCs in the LPS-induced ALI model. The left lungs from mice of different groups were collected $24 \mathrm{~h}$ after LPS intervention, and the prepared lung MNCs were stained for CD11c and F4/80 and analyzed by flow cytometry. DCs were marked as

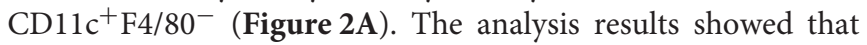
the positive expression percentage of MHCII, CD80, CD86, and CD40 was significantly increased in the ALI group in contrast to those in the control, and the increase in MHCII, CD86, and CD40 was further augmented by rHMGB1 treatment. By contrast, the increase in MHCII, CD80, CD86, and CD40 was obviously lowered in the ALI group treated with anti-HMGB1 (Figures 2B,C). Subsequently, we observed the effect of HMGB1 on proinflammatory cytokines released by LPS-primed BMDCs in vitro. The levels of TNF- $\alpha$, IL-6, IL-18, IL-1 $\beta$, MCP-1, and IL-12 cytokines released by DCs, which reflect the maturation of DCs, were significantly upregulated by LPS stimulation in the BMDC culture supernatant compared with those in the control group. On the other hand, rHMGB1 stimulation also significantly increased the production of these proinflammatory 


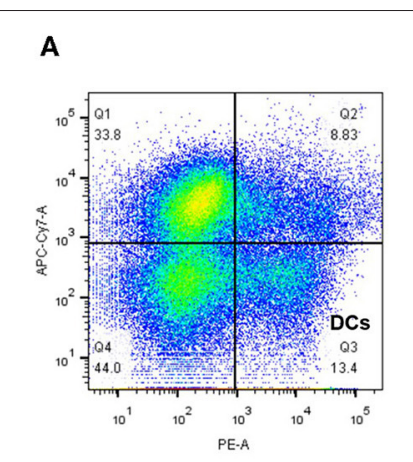

B
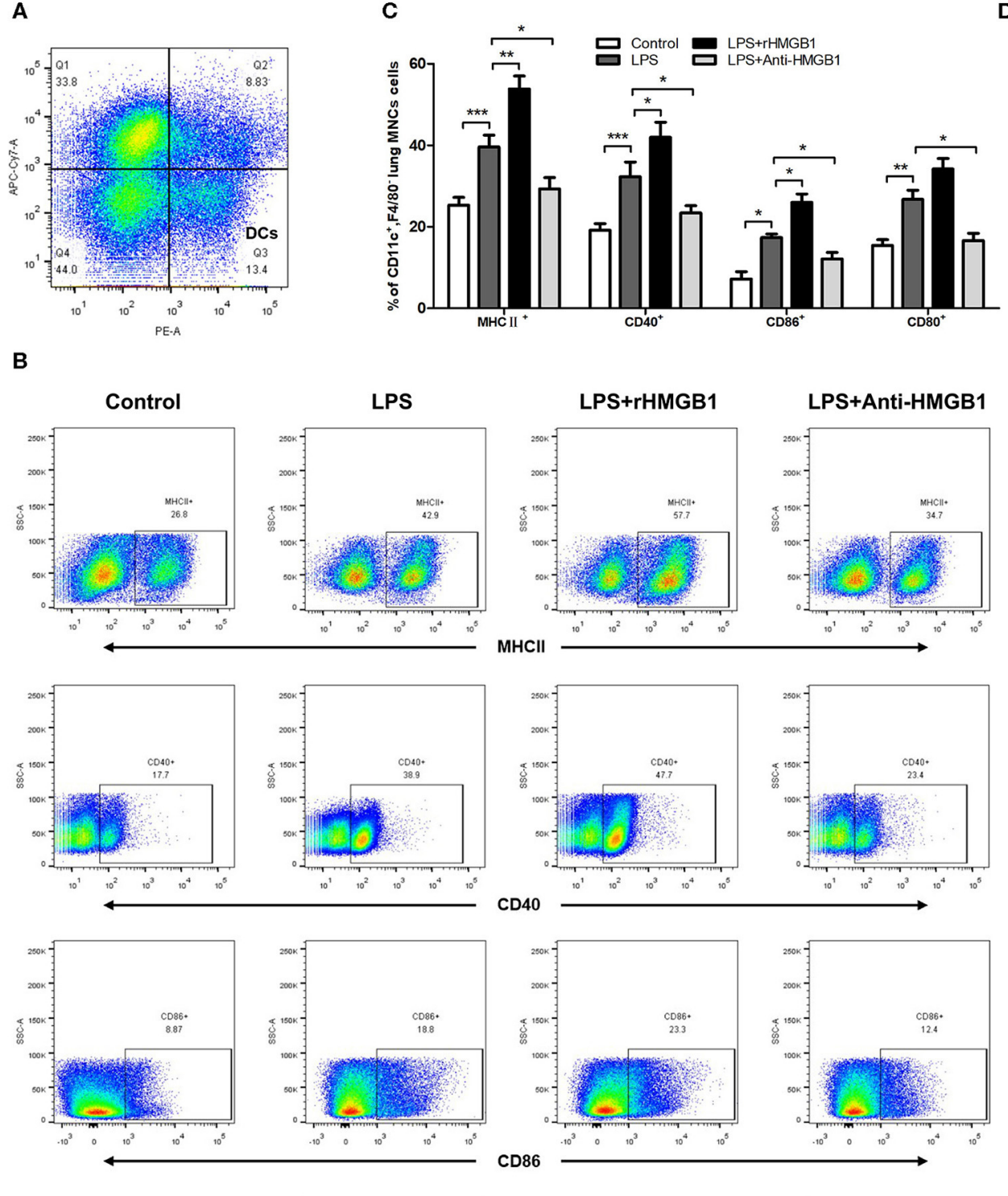

D
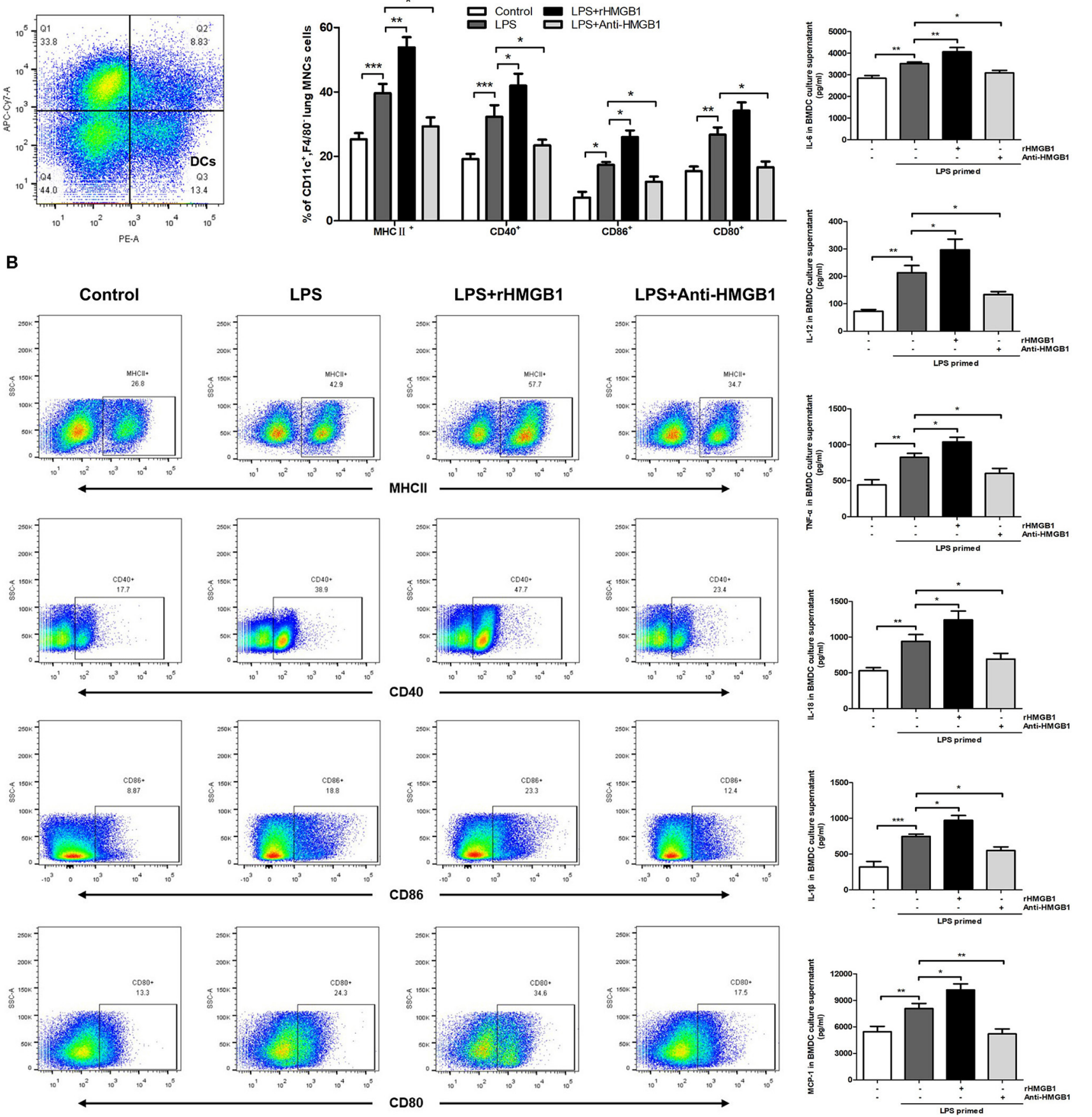

FIGURE 2 | Anti-HMGB1 and rHMGB1 regulated the maturation of DCs in vivo and in vitro. (A) Lung DCs were stained as CD11 ${ }^{+}$F4/80- . (B,C) Positive expression percentage of MHCII, CD80, CD86, and CD40 was measured in DCs (CD11C+F4/80-) by flow cytometric analysis. (D) The levels of cytokines TNF- $\alpha$, IL-6, IL-18, $\mathrm{IL}-1 \beta, \mathrm{MCP}-1$, and IL-12 secretion in BMDC culture supernatants were measured by ELISA. ${ }^{*} p<0.05,{ }^{* *} p<0.01,{ }^{* * *} p<0.001$.

cytokines. Opposite results were observed in the groups receiving anti-HMGB1 stimulation (Figure 2D). These data suggested that HMGB1 induces the maturation and enhances the antigenpresenting ability of DCs in the ALI mice model and in LPSprimed BMDCs.

\section{HMGB1 Affected the Phenotypic Changes of DCs in ALI Mice Model}

Subsequently, we observed the effect of anti-HMGB1 and rHMGB1 on phenotypic and functional changes in the DCs of lung tissue from the LPS-induced ALI mice model. While 
detecting intercellular adhesion molecule 1 (ICAM-1), CD11b (a marker for myeloid dendritic cells [mDCs]), B220 (a marker of plasmacytoid dendritic cells [pDCs]), and chemotactic factor CCR7 in the surface of lung DCs by flow cytometric analysis, we found that the percentage of ICAM-1, CD11b, or CCR7-positive expression was significantly elevated in the LPS-administrated ALI group in contrast to the control. The increase in ICAM-1, CD11b, and CCR7 percentage was significantly higher in the rHMGB1-treated ALI group relative to those of the ALI group. In addition, the increase in ICAM-1, CD11b, and CCR7 was reduced after anti-HMGB1 treatment (Figures 3A,B). However, the percentage of B220positive expression among different groups was not significantly different (Figures 3A,B). These data demonstrated that HMGB1 upregulated the percentage of $\mathrm{mDCs}$ and enhanced the adhesion and chemotaxis of DCs.

\section{Inhibition of PI3K/Akt/mTOR Signaling Pathway Weakens the Maturation of DCs in vivo and in vitro}

We next verified whether the PI3K/Akt/mTOR signaling pathway affects the maturation and function of DCs in the presence of a PI3K inhibitor (LY294002) in vivo and in vitro. Flow cytometric analysis in vivo showed that the percentage of $\mathrm{MHCII}^{+}, \mathrm{CD}^{+}$, $\mathrm{CD}^{+} 6^{+}$, or $\mathrm{CD}_{4}{ }^{+} \mathrm{DCs}$ was significantly higher in ALI mice compared with control mice. A significant decrease was also observed in the percentage of $\mathrm{MHCII}^{+}, \mathrm{CD}^{+} 0^{+} \mathrm{CD}^{+}{ }^{+}$, and $\mathrm{CD}_{4}{ }^{+}$DCs in the LY294002-treated mice compared with that in the LPS-induced ALI mice (Figures 4A,B). In vitro experiments with BMDCs revealed that stimulation with LPS significantly augmented the levels of TNF- $\alpha$, IL-6, IL-18, IL$1 \beta$, MCP-1, and IL-12 in the culture supernatant. The levels of these proinflammatory cytokines produced by BMDCs were reduced with the inhibition of PI3K (in the LPS+LY294002 group) (Figure 4C). Thus, inhibiting the PI3K/Akt/mTOR signaling pathway attenuated the mature differentiation of DCs in ALI.

\section{PI3K/Akt/mTOR Signaling Pathway Affected the Phenotypic Changes of DCs in ALI Mice Model}

To demonstrate the role of the PI3K/Akt/mTOR signaling pathway in phenotypic and functional changes of DCs in the ALI mice model, we also treated LPS-induced mice with LY294002 and detected the percentage of ICAM-1, CD11b, B220, and CCR7 in lung DCs by flow cytometric analysis. In comparison with the control group, LPS-induced ALI groups showed a significant increase in the percentage of DCs $\left(\mathrm{CD} 11 \mathrm{c}^{+} \mathrm{F} 4 / 80^{-} \mathrm{MNCs}\right)$ expressing ICAM-1, CD11b, and CCR7. These changes were weakened with LY294002 treatment. In addition, no significant difference arose in the percentage of B220 between different groups (Figures 5A,B). These results suggested that, similarly to HMGB1, the PI3K/Akt/mTOR signaling pathway also influenced the percentage of $\mathrm{mDCs}$ and mediated the adhesion and chemotactic ability of DCs to T cells.

\section{DISCUSSION}

In the current study, we show that HMGB1 activates the PI3K/Akt/mTOR signaling pathway in BMDCs and upregulates the expression of PI3K, Akt, and mTOR mRNA and corresponding phosphorylated proteins. HMGB1 and the $\mathrm{PI} 3 \mathrm{~K} / \mathrm{Akt} / \mathrm{mTOR}$ pathway form the HMGB1-PI3K/Akt/mTOR signaling pathway in lung DCs, and this pathway then induces the maturation and antigen-presenting ability of lung DCs while also mediating the percentage of mDCs and enhancing the adhesion and chemotactic ability of lung DCs.

HMGB1 functions to regulate the innate immune system. Recent studies have shown that as a late proinflammatory cytokine, HMGB1 has a key role in the pathological progress of ALI and regulates the lung inflammatory response $(4,5)$. HMGB1 is an upstream mediator of TLR2 and TLR4, the main receptors of HMGB1. Together these form the HMGB1-TLR2, TLR4 pathway, which contributes to inflammatory response via multiple mechanisms (6-8). Besides activating the NF- $\kappa$ B and MAPK pathways, recent studies also have demonstrated that HMGB1 regulates the PI3K/Akt/mTOR signaling pathway in myocardial ischemia/reperfusion injury and LPS-induced pulmonary inflammation in ALI models $(9,10,13,14)$. The PI3K/AKT/mTOR signaling pathway has been shown to contribute to the regulation of cell survival during oxidative stress and to participate in the pulmonary inflammatory progression of ALI (11-14). These studies agree with our results for the relationship between HMGB1 and the PI3K/Akt/mTOR pathway in DCs during ALI.

As specialized antigen-presenting cells pivotal for the initial and adaptive immune response, DCs are outpost cells of immune defense in the respiratory system. Under the stimulation of pathogens, DCs are activated and then mature and migrate to lymph nodes (30). These mature and activated DCs upregulate the surface expression of MHCII and diverse costimulatory molecules (CD80, CD86, and CD40) and release inflammatory cytokines, which train and stimulate Th cells to differentiate into various subtypes (28-30). DCs regulate acute lung inflammation and injury in LPS-induced ALI, and mature DCs participate in aggravating acute lung tissue injury and inflammatory response $(17,18)$.

In humans and mice, $\mathrm{DCs}$ have two important subsets: mDCs $\left(\mathrm{CD} 11 \mathrm{c}^{+} \mathrm{F} 4 / 80^{-} \mathrm{CD} 11 \mathrm{~b}^{+}\right)$and $\mathrm{pDCs}\left(\mathrm{CD} 11 \mathrm{c}^{+} \mathrm{F} 4 / 80^{-} \mathrm{B} 220^{+}\right)$. Compared with pDCs, the mDC subset displays high expression levels of costimulatory molecules CD80, CD86, CD40, and MHCII and also better stimulate the proliferation and differentiation of $\mathrm{T}$ cells (31). This indicates that the maturity of pDCs is inferior to that of mDCs. Moreover, pDCs do not express LPS-specific receptors TLR2 and TLR4 and therefore cannot be stimulated to maturation by LPS (31). In addition, DCs also express an important adhesive molecule on their cell surface, ICAM-1, which mediates adhesion reaction, and release an important chemokine, CCR7, which has the ability to induce directional chemotaxis (32-34). In our present study, we detected the expression of surface markers (CD80, CD86, CD40, MHC II, B220, CD11b, ICAM-1, CCR7) to reflect the 
A
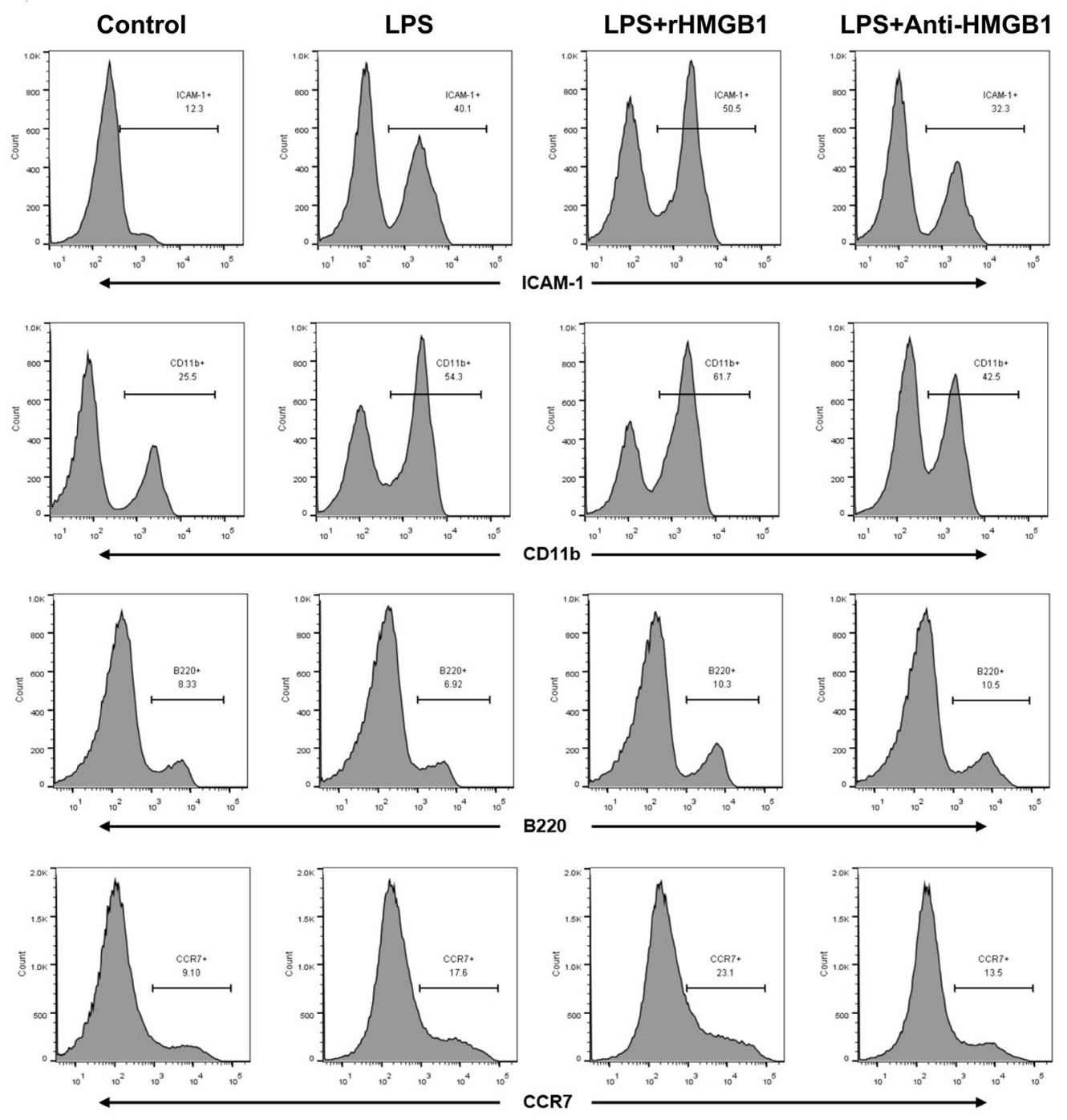

B

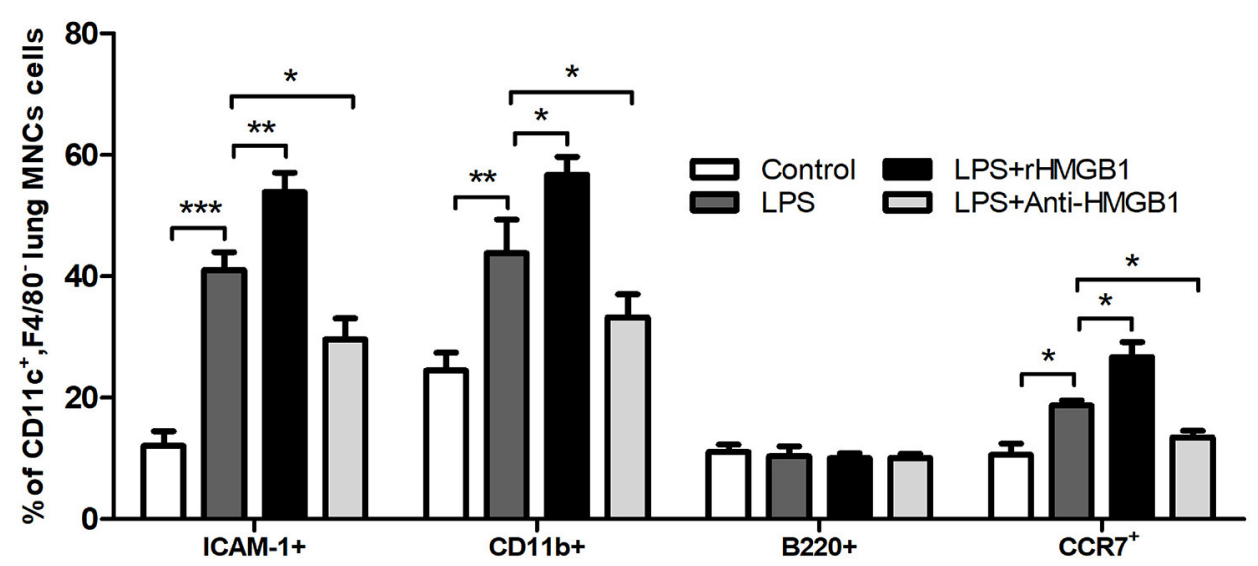

FIGURE 3 | Anti-HMGB1 and rHMGB1 affected the phenotype and function of DCs. (A,B) Positive expression percentage of ICAM-1, CD11b, B220, and CCR7 was measured in DCs $\left(\mathrm{CD} 11 \mathrm{C}^{+} \mathrm{F} 4 / 80^{-}\right)$by flow cytometric analysis. ${ }^{\star} p<0.05,{ }^{* *} p<0.01,{ }^{* \star} p<0.001$. 
A
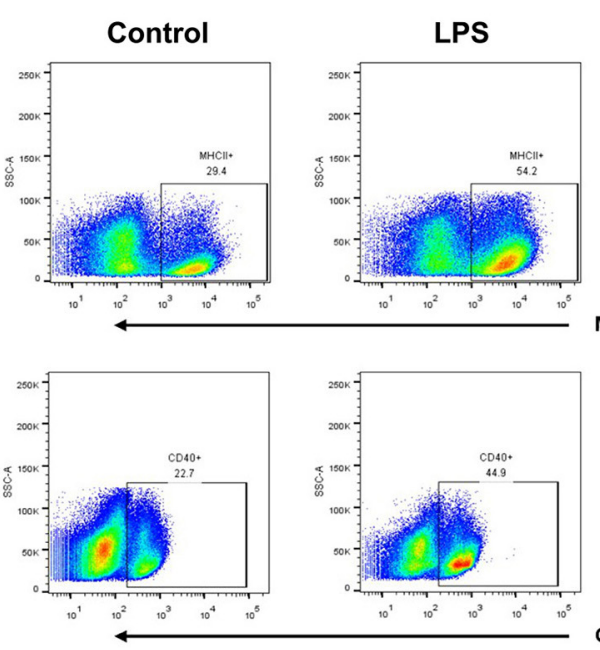

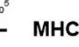
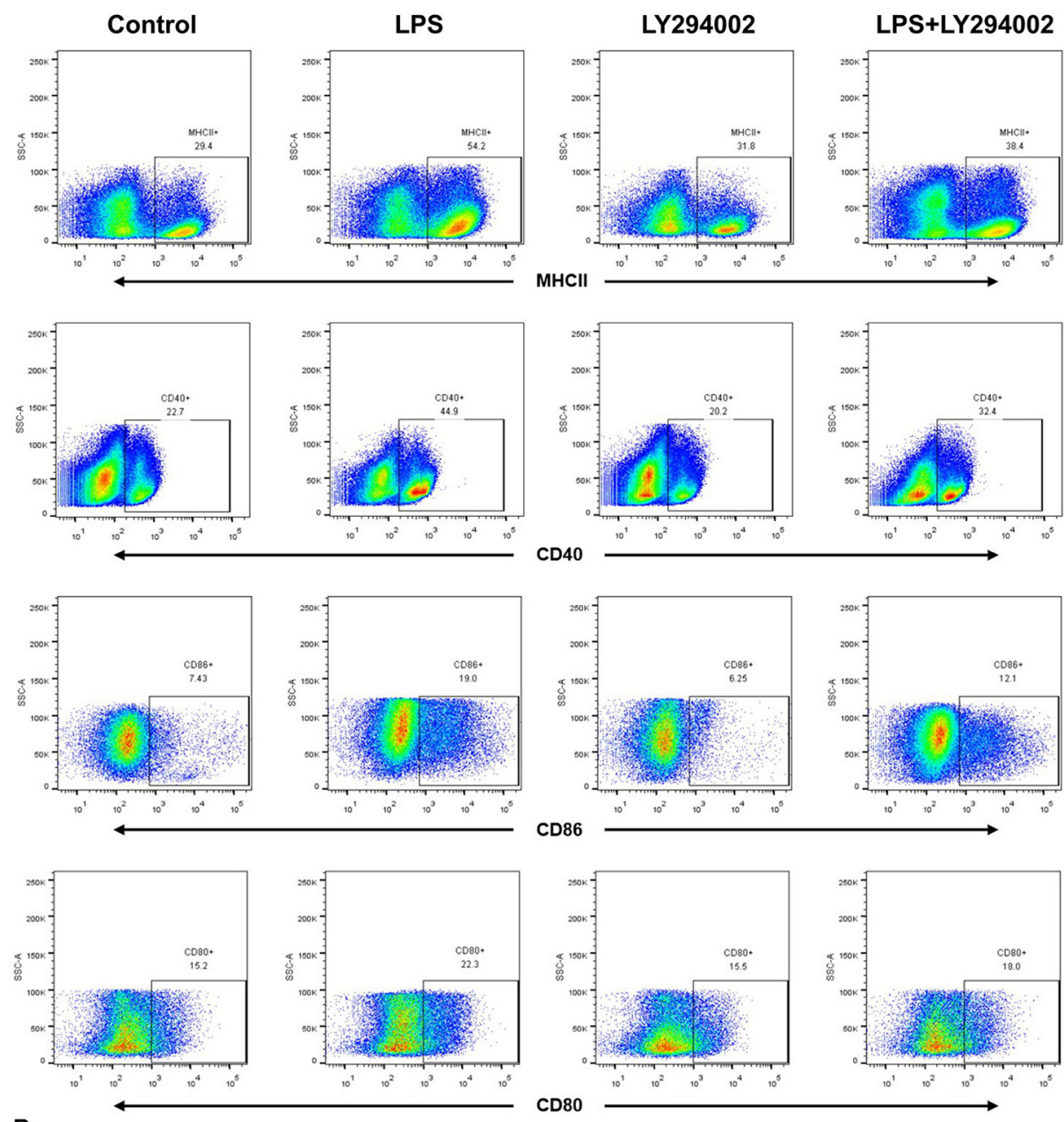

B

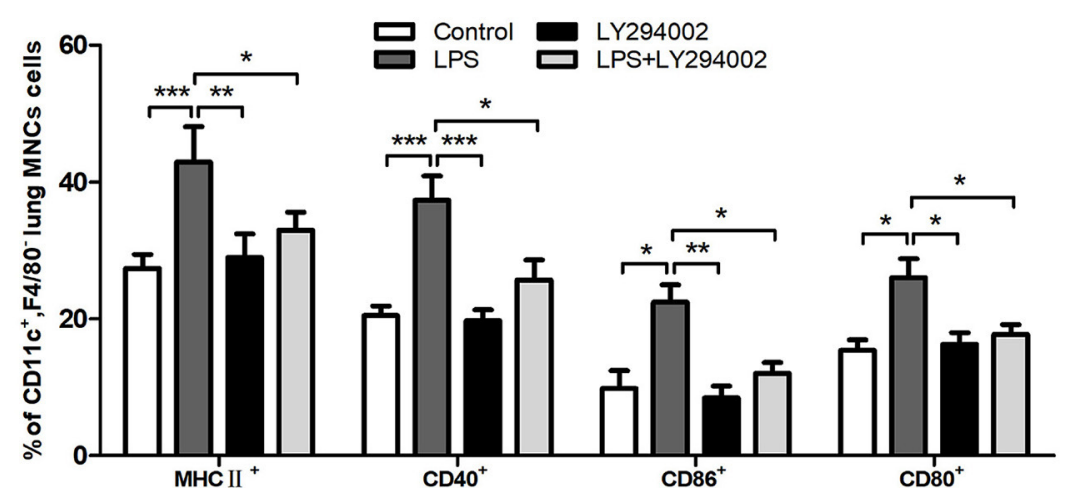

C
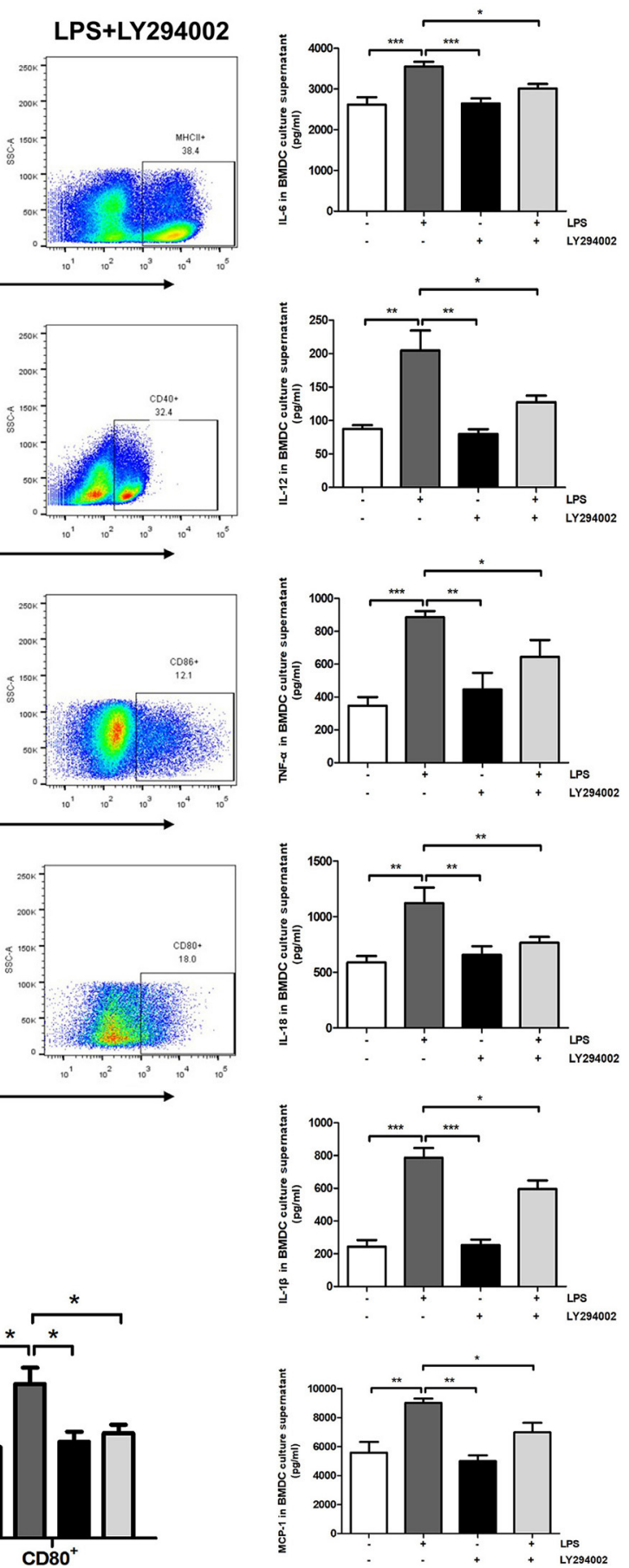

FIGURE 4 | Inhibition of PI3K by LY294002 regulated the maturation of DCs in vivo and in vitro. (A,B) Positive expression percentage of MHCII, CD80, CD86, and CD40 was measured in DCs (CD11 ${ }^{+} F 4 / 80^{-}$) by flow cytometric analysis. (C) Levels of secreted cytokines TNF- $\alpha$, IL-6, IL-18, IL-1 $\beta$, MCP-1, and IL-12 in BMDCs culture supernatant were measured by ELISA. ${ }^{\star} p<0.05,{ }^{* \star} p<0.01,{ }^{* \star *} p<0.001$.

effect of the HMGB1/PI3K/Akt/mTOR signaling pathway on the phenotype and function of DCs in ALI. Our results showed that the HMGB1/PI3K/Akt/mTOR signaling pathway induced upregulation of markers CD80, CD86, CD40, MHC II, CD11b,
ICAM-1, and CCR7, suggesting that the proportion of mature DCs increased; the ratio of mDCs also increased, accompanied by the augmentation of antigen presentation, adhesion, and chemotactic ability. 
A
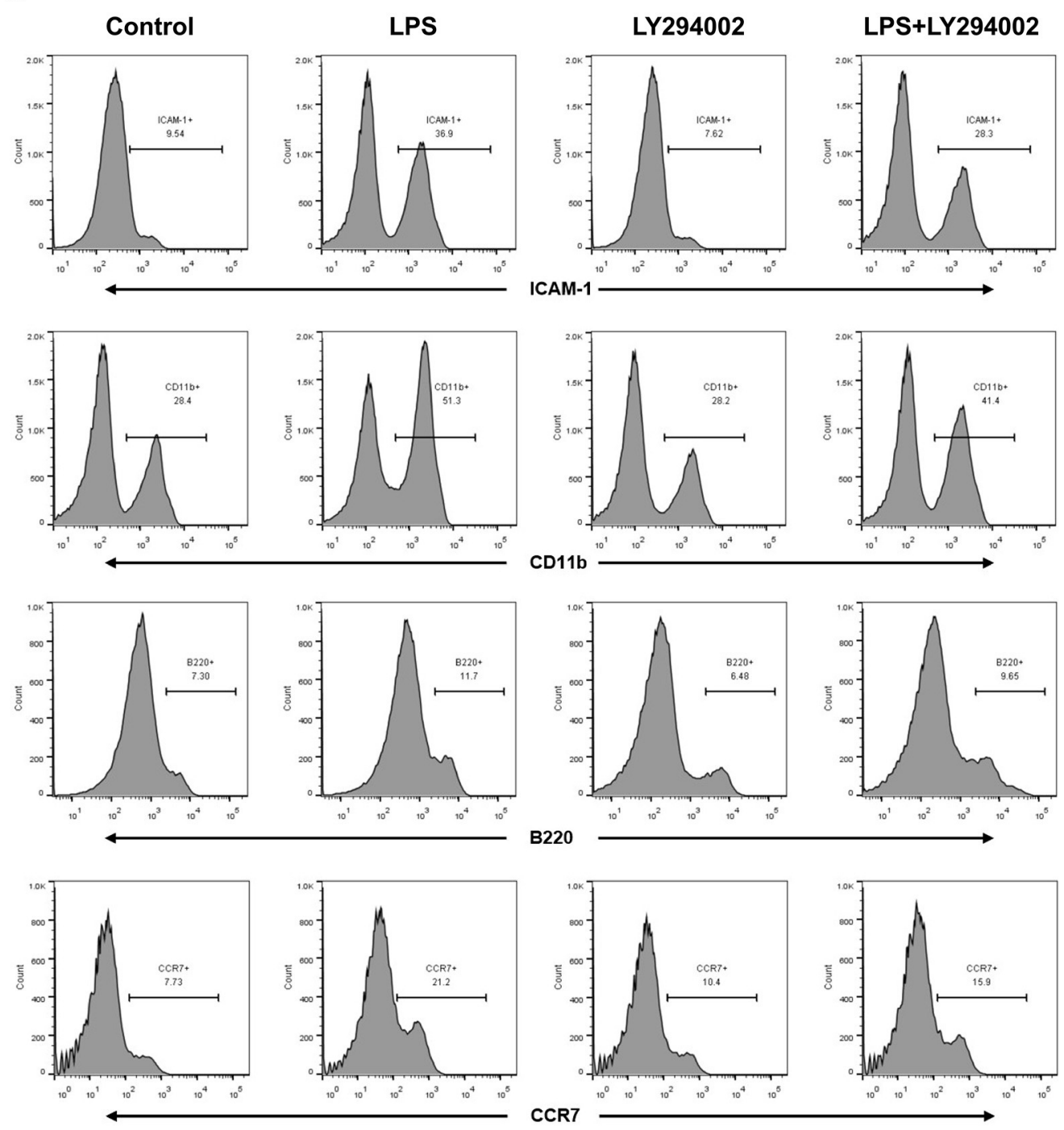

B

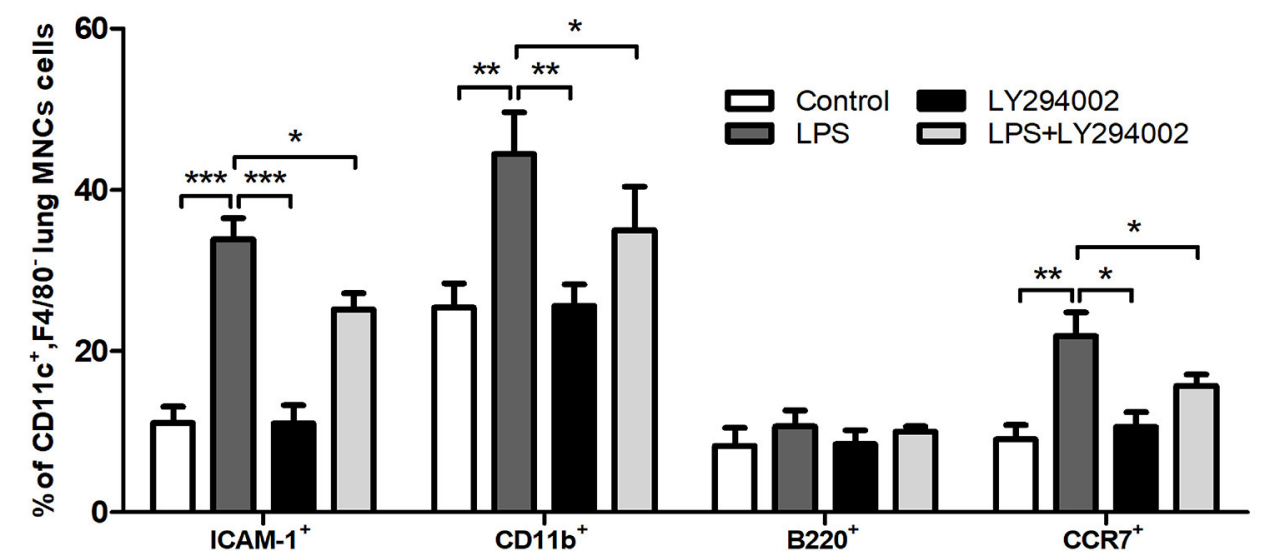

FIGURE 5 | Inhibition of PI3K by LY294002 affected the phenotype and function of DCs. (A,B) Positive expression percentage of ICAM-1, CD11b, B220, and CCR7 was measured in DCs $\left(\mathrm{CD} 11 \mathrm{C}^{+} \mathrm{F} 4 / 80^{-}\right)$by flow cytometric analysis. ${ }^{\star} p<0.05,{ }^{* \star} p<0.01,{ }^{* \star *} p<0.001$. 
In conclusion, our present study provides evidence of the role of the HMGB1/PI3K/Akt/mTOR signaling pathway at the level of DCs in ALI and further confirms that HMGB1/PI3K/Akt/mTOR signaling participates in the pathological process of ALI by regulating the maturation and functions of DCs.

\section{DATA AVAILABILITY STATEMENT}

The datasets presented in this article are not readily available because access to this dataset is restricted. Requests to access the datasets should be directed to 498676772@qq.com.

\section{REFERENCES}

1. Matthay MA, Ware LB, Zimmerman GA. The acute respiratory distress syndrome. J Clin Invest. (2012) 122:2731-40. doi: 10.1172/JCI60331

2. Fan E, Brodie D, Slutsky AS. Acute respiratory distress syndrome advances in diagnosis and treatment. JAMA. (2018) 319:698-710. doi: 10.1001/jama.2017.21907

3. Bellani G, Laffey JG, Pham T, Fan E, Brochard L, Esteban A, et al. Epidemiology, patterns of care, and mortality for patients with acute respiratory distress syndrome in intensive care units in 50 countries. JAMA. (2016) 315:788-800. doi: 10.1001/jama.2016.0291

4. Li N, Liu XX, Hong M, Huang XZ, Chen H, Xu JH, et al. Sodium butyrate alleviates LPS-induced acute lung injury in mice via inhibiting HMGB1 release. Int Immunopharmacol. (2018) 56:242-8. doi: 10.1016/j.intimp.2018.01.017

5. Song JH, Kim JY, Piao C, Lee S, Kim B, Song SJ, et al. Delivery of the high-mobility group box 1 box A peptide using heparin in the acute lung injury animal models. J Control Release. (2016) 234:33-40. doi: 10.1016/j.jconrel.2016.05.039

6. Di Candia L, Gomez E, Venereau E, Chachi L, Kaur D, Bianchi ME, et al. HMGB1 is upregulated in the airways in asthma and potentiates airway smooth muscle contraction via TLR4. J Allergy Clin Immunol. (2017) 140:5847. doi: 10.1016/j.jaci.2016.11.049

7. Achouiti A, van der Meer AJ, Florquin S, Yang H, Tracey KJ, van 't Veer C, et al. High-mobility group box 1 and the receptor for advanced glycation end products contribute to lung injury during Staphylococcus aureus pneumonia. Crit Care. (2013) 17:R296. doi: 10.1186/cc13162

8. He ZW, Qin YH, Wang ZW, Chen Y, Shen Q, Dai SM. HMGB1 acts in synergy with lipopolysaccharide in activating rheumatoid synovial fibroblasts via p38 MAPK and NF-kappaB signaling pathways. Mediators Inflamm. (2013) 2013:596716. doi: 10.1155/2013/596716

9. Meng L, Li L, Lu S, Li K, Su Z, Wang Y, et al. The protective effect of dexmedetomidine on LPS-induced acute lung injury through the HMGB1-mediated TLR4/NF- $\mathrm{B}$ and PI3K/Akt/mTOR pathways. Mol Immunol. (2018) 94:7-17. doi: 10.1016/j.molimm.2017. 12.008

10. Li X, Hu X, Wang J, Xu W, Yi C, Ma R, et al. Short-term hesperidin pretreatment attenuates rat myocardial ischemia/reperfusion injury by inhibiting high mobility group box 1 protein expression via the PI3K/Akt pathway. Cell Physiol Biochem. (2016) 39:1850-62. doi: 10.1159/000447884

11. Sui H, Luo M, Miao Y, Cheng W, Shan W, Zhao B, et al. Cystic fibrosis transmembrane conductance regulator ameliorates lipopolysaccharideinduced acute lung injury by inhibiting autophagy through PI3K/AKT/mTOR pathway in mice. Respir Physiol Neurobiol. (2019) 273:103338. doi: 10.1016/j.resp.2019.103338

12. Feng FB, Qiu HY. Effects of Artesunate on chondrocyte proliferation, apoptosis and autophagy through the PI3K/AKT/mTOR signaling pathway in rat models with rheumatoid arthritis. Biomed Pharmacother. (2018) 102:120920. doi: 10.1016/j.biopha.2018.03.142

\section{ETHICS STATEMENT}

The animal study was reviewed and approved by Huazhong University of Science and Technology.

\section{AUTHOR CONTRIBUTIONS}

RL and YS designed the research. RL, XZ, YY, HZ, PL, SP, and $\mathrm{YO}$ performed the experiments. RL analyzed the data and produced the figures. RL, HH, and YS wrote the manuscript. All authors contributed to the article and approved the submitted version.

13. Li R, Ren T, Zeng J. Mitochondrial coenzyme q protects sepsis-induced acute lung injury by activating PI3K/Akt/GSK-3 $\beta / \mathrm{mTOR}$ pathway in rats. Biomed Res Int. (2019) 2019:5240898. doi: 10.1155/2019/5240898

14. Qu L, Chen C, He W, Chen Y, Li Y, Wen Y, et al. Glycyrrhizic acid ameliorates LPS-induced acute lung injury by regulating autophagy through the PI3K/AKT/mTOR pathway. Am J Transl Res. (2019) 11:2042-55.

15. Kopf M, Schneider C, Nobs SP. The development and function of lungresident macrophages and dendritic cells. Nat Immunol. (2015) 16:36-44. doi: 10.1038/ni.3052

16. Yi S, Zhai J, Niu R, Zhu G, Wang M, Liu J, et al. Eosinophil recruitment is dynamically regulated by interplay among lung dendritic cell subsets after allergen challenge. Nat Commun. (2018) 9:3879. doi: 10.1038/s41467-018-06316-9

17. Lu Z, Chang W, Meng S, Xu X, Xie J, Guo F, et al. Mesenchymal stem cells induce dendritic cell immune tolerance via paracrine hepatocyte growth factor to alleviate acute lung injury. Stem Cell Res Ther. (2019) 10:372. doi: 10.1186/s13287-019-1488-2

18. Li L, Dong L, Zhao D, Gao F, Yan J. Classical dendritic cells regulate acute lung inflammation and injury in mice with lipopolysaccharide-induced acute respiratory distress syndrome. Int J Mol Med. (2019) 44:617-29. doi: 10.3892/ijmm.2019.4208

19. Ding J, Feng T, Ning Y, Li W, Wu Q, Qian K, et al. $\beta$-Glucan enhances cytotoxic $\mathrm{T}$ lymphocyte responses by activation of human monocyte-derived dendritic cells via the PI3K/AKT pathway. Hum Immunol. (2015) 76:146-54. doi: 10.1016/j.humimm.2015.01.009

20. Xuan NT, Hoang NH, Nhung VP, Duong NT, Ha NH, Hai NV. Regulation of dendritic cell function by insulin/IGF-1/PI3K/Akt signaling through klotho expression. J Recept Signal Transduct Res. (2017) 37:297-303. doi: 10.1080/10799893.2016.1247862

21. de Oliveira MTP, de Sá Coutinho D, Tenório de Souza É, Stanisçuaski Guterres S, Pohlmann AR, Silva PMR, et al. Orally delivered resveratrolloaded lipid-core nanocapsules ameliorate LPS-induced acute lung injury via the ERK and PI3K/Akt pathways. Int J Nanomedicine. (2019) 14: 5215-28. doi: $10.2147 / \mathrm{IJN}$.S200666

22. Li R, Shang Y, Yu Y, Zhou T, Xiong W, Zou X. High-mobility group box 1 protein participates in acute lung injury by activating protein kinase R and inducing M1 polarization. Life Sci. (2020) 6:117415. doi: 10.1016/j.lfs.2020.117415

23. Gao M, Ha T, Zhang X, Wang X, Liu L, Kalbfleisch J, et al. The Toll-like receptor 9 ligand, $\mathrm{CpG}$ oligodeoxynucleotide, attenuates cardiac dysfunction in polymicrobial sepsis, involving activation of both phosphoinositide 3 kinase/Akt and extracellular-signal-related kinase signaling. J Infect Dis. (2013) 207:1471-9. doi: 10.1093/infdis/jit036

24. Li R, Wang J, Li R, Zhu F, Xu W, Zha G, et al. ATP/P2X7-NLRP3 axis of dendritic cells participates in the regulation of airway inflammation and hyper-responsiveness in asthma by mediating HMGB1 expression and secretion. Exp Cell Res. (2018) 366:1-15. doi: 10.1016/j.yexcr.2018.03.002

25. Takáč T, Pechan T, Samajová O, Šamaj J. Vesicular trafficking and stress response coupled to PI3K inhibition by LY294002 as revealed by 
proteomic and cell biological analysis. J Proteome Res. (2013) 12:4435-48. doi: 10.1021/pr400466x

26. Li R, Wang J, Zhu F, Li R, Liu B, Xu W, et al. HMGB1 regulates T helper 2 and $\mathrm{T}$ helper17 cell differentiation both directly and indirectly in asthmatic mice. Mol Immunol. (2018) 97:45-55. doi: 10.1016/j.molimm.2018. 02.014

27. Liu H, Liu W, Qiu H, Zou D, Cai H, Chen Q, et al. Salvianolic acid B protects against myocardial ischaemia-reperfusion injury in rats via inhibiting high mobility group box 1 protein expression through the PI3K/Akt signalling pathway. Naunyn-Schmiedeberg's Arch Pharmacol. (2019). doi: 10.1007/s00210-019-01755-7. [Epub ahead of print].

28. Yang L, Han X, Yuan J, Xing F, Hu Z, Huang F, et al. Early astragaloside IV administration attenuates experimental autoimmune encephalomyelitis in mice by suppressing the maturation and function of dendritic cells. Life Sci. (2020) 19:117448. doi: 10.1016/j.lfs.2020.117448

29. Sauter A, Yi DH, Li Y, Roersma S, Appel S. The culture dish surface influences the phenotype and cytokine production of human monocyte-derived dendritic cells. Front Immunol. (2019) 10:2352. doi: 10.3389/fimmu.2019.02352

30. Ahad A, Stevanin M, Smita S, Mishra GP, Gupta D, Waszak S, et al. NCoR1: putting the brakes on the dendritic cell immune tolerance. iScience. (2019) 19:996-1011. doi: 10.1016/j.isci.2019.08.024

31. Gulubova M. Myeloid and plasmacytoid dendritic cells and cancernew insights. Open Access Maced J Med Sci. (2019) 7:3324-40. doi: 10.3889/oamjms.2019.735
32. Comrie WA, Li S, Boyle S, Burkhardt JK. The dendritic cell cytoskeleton promotes $\mathrm{T}$ cell adhesion and activation by constraining ICAM-1 mobility. J Cell Biol. (2015) 208:457-73. doi: 10.1083/jcb.201406120

33. Haghayegh Jahromi N, Marchetti L, Moalli F, Duc D, Basso C, Tardent H, et al. Intercellular adhesion molecule-1 (ICAM-1) and ICAM-2 differentially contribute to peripheral activation and CNS entry of autoaggressive Th1 and Th17 cells in experimental autoimmune encephalomyelitis. Front Immunol. (2020) 10:3056. doi: 10.3389/fimmu.2019.03056

34. Hammerschmidt SI, Werth K, Rothe M, Galla M, Permanyer M, Patzer GE, et al. CRISPR/Cas9 immunoengineering of Hoxb8-immortalized progenitor cells for revealing CCR7-mediated dendritic cell signaling and migration mechanisms in vivo. Front Immunol. (2018) 9:1949. doi: 10.3389/fimmu.2018.01949

Conflict of Interest: The authors declare that the research was conducted in the absence of any commercial or financial relationships that could be construed as a potential conflict of interest.

Copyright (c) 2020 Li, Zou, Huang, Yu, Zhang, Liu, Pan, Ouyang and Shang. This is an open-access article distributed under the terms of the Creative Commons Attribution License (CC BY). The use, distribution or reproduction in other forums is permitted, provided the original author(s) and the copyright owner(s) are credited and that the original publication in this journal is cited, in accordance with accepted academic practice. No use, distribution or reproduction is permitted which does not comply with these terms. 